\title{
Status and future work of the NEA Working Party on international nuclear data Evaluation Cooperation
}

\author{
Arjan J. Koning ${ }^{1}$, Jun-ichi Katakura ${ }^{2}$, Pavel Oblozinsky ${ }^{3}$, Alan L. Nichols ${ }^{4}$, and Claes Nordborg ${ }^{5}$ \\ ${ }^{1}$ NRG, 3 Westerduinweg, P.O. Box 25, 1755 ZG Petten, The Netherlands \\ 2 Nuclear Data Center, Japan Atomic Energy Agency, Tokai-mura, Naka-gun, Ibaraki-ken 319-1195, Japan \\ 3 National Nuclear Data Center, Brookhaven National Laboratory, P.O. Box 5000, Upton, NY 11973-5000, USA \\ ${ }^{4}$ Nuclear Data Section, International Atomic Energy Agency, P.O. Box 100, Wagramer Strasse 5, 1400 Vienna, Austria \\ 5 OECD Nuclear Energy Agency, 12 bd des îles, 92130 Issy-les-Moulineaux, France
}

\begin{abstract}
The Working Party on international nuclear data Evaluation Cooperation (WPEC) is a collaborative effort between the major OECD nuclear data projects: ENDF/B (USA), JENDL (Japan) and JEFF (Europe), and the IAEAsupported non-OECD projects CENDL (China) and BROND (Russian Federation). Nuclear data challenges and problems that are common to all projects are considered in various WPEC subgroups that are normally of limited time span. The achievements of these subgroups during the course of 2005/06 are reviewed in this paper. Among the recent accomplishments are improved criticality predictions through revised $U$ evaluations, the completion of new cross-section standards, and the creation of a new fission product library. Problems that are currently being addressed are the processing and inclusion of covariance data in the libraries, improvements in decay data to assist in decay heat calculations, assembly of a well-structured High Priority Request List, validation of the new fission product cross-section library, and the assessment of nuclear data needs for GEN-IV.
\end{abstract}

\section{Introduction}

The OECD Nuclear Energy Agency (NEA) established the Working Party on international nuclear data Evaluation Cooperation (WPEC) in 1989 to provide a framework for cooperative activities between the major nuclear data evaluation projects. The collaboration started between the US ENDF, the European JEF and the Japanese JENDL projects and was subsequently extended to include the Russian BROND, the Chinese CENDL and the IAEA FENDL projects through the support of the IAEA.

The main objectives of the cooperation are as follows:

- improve the quality and completeness of the evaluated nuclear data libraries by means of international cooperation,

- coordinate the nuclear data measurements required within this framework.

The WPEC assesses the needs for improvements in nuclear data, and addresses these needs by setting up subgroups to perform agreed technical co-operative activities. So far this form of cooperation has resulted in the completion of 21 studies covering many different issues, from generic topics, such as delayed-neutron data and intermediate energy data, to specific topics, such as the epithermal capture of ${ }^{235} \mathrm{U}$.

The outcome and recommendations of the WPEC subgroups are published in reports (fig. 1) that can be obtained from the NEA or downloaded directly from the WPEC homepage at http://www.nea.fr/html/science/wpec/.

\section{Recent achievements}

The WPEC completed six studies in 2005/06, as described below, of which five have been published.

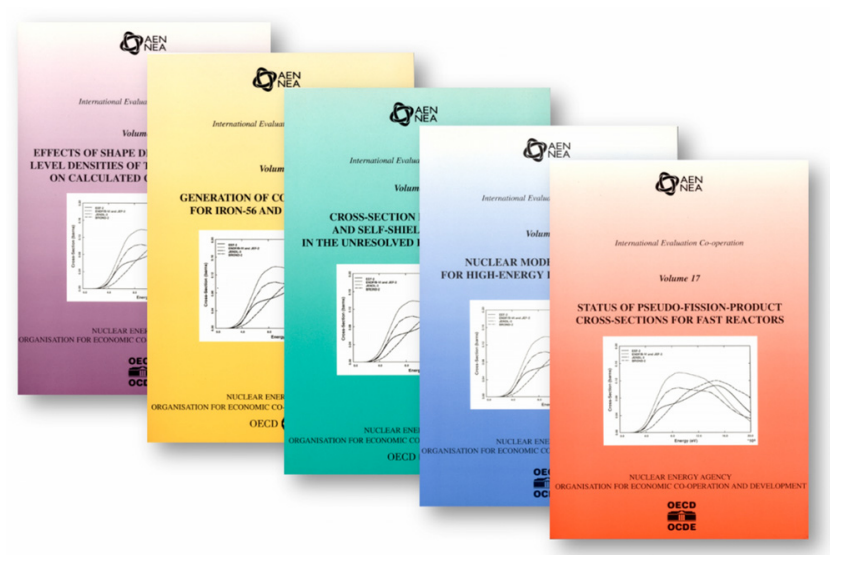

Fig. 1. Examples of WPEC reports.

\subsection{Nuclear data standards}

The decision to re-evaluate the nuclear data standards was based on a general consensus that these data needed to be updated (see table 1). There have been significant improvements to the experimental database since 1987, when the previous comprehensive evaluation was completed. The work was performed by means of an efficient collaboration between a WPEC subgroup [1], a task force of the US Cross Section Evaluation Working Group (CSEWG), and a Coordinated Research Project of the International Atomic Energy Agency (IAEA).

\subsection{Activation cross sections}

As the need for improved model calculations became apparent from measurements of reaction cross-sections by means of 
Table 1. Neutron cross section standards.

\begin{tabular}{ll}
\hline Reaction & Standards energy range \\
\hline $\mathrm{H}(\mathrm{n}, \mathrm{n})$ & $1 \mathrm{keV}$ to $20 \mathrm{MeV}$ \\
${ }^{3} \mathrm{He}(\mathrm{n}, \mathrm{p})$ & $0.0253 \mathrm{eV}$ to $50 \mathrm{keV}$ \\
${ }^{6} \mathrm{Li}(\mathrm{n}, \mathrm{t})$ & $0.0253 \mathrm{eV}$ to $1 \mathrm{MeV}$ \\
${ }^{10} \mathrm{~B}(\mathrm{n}, \alpha)$ & $0.0253 \mathrm{eV}$ to $1 \mathrm{MeV}$ \\
${ }^{10} \mathrm{~B}\left(\mathrm{n}, \alpha_{1} \gamma\right)$ & $0.0253 \mathrm{eV}$ to $1 \mathrm{MeV}$ \\
$\mathrm{C}(\mathrm{n}, \mathrm{n})$ & $0.0253 \mathrm{eV}$ to $1.8 \mathrm{MeV}$ \\
$\mathrm{Au}(\mathrm{n}, \gamma)$ & $0.0253 \mathrm{eV}, 0.2$ to $2.5 \mathrm{MeV}$ \\
${ }^{235} \mathrm{U}(\mathrm{n}, \mathrm{f})$ & $0.0253 \mathrm{eV}, 0.15$ to $200 \mathrm{MeV}$ \\
${ }^{238} \mathrm{U}(\mathrm{n}, \mathrm{f})$ & $2 \mathrm{MeV}$ to $200 \mathrm{MeV}$ \\
\hline
\end{tabular}

the activation technique, the WPEC set up an appropriate subgroup [3]. The main objectives were to validate the nuclear modelling by benchmark calculations based on new and recent measurements. The following tasks were undertaken:

- generation of a large amount of pertinent experimental nuclear data to validate the nuclear models,

- determination of nuclear model parameters that yield the best agreement with experimental data,

- provision of reliable evaluations for a large number of neutron activation reactions,

- nuclear-model sensitivity studies for specific reactions.

A detailed description of the evaluation procedures and the resulting nuclear data standards can be found in a paper presented at this conference [2].

Activation data from a large number of measurements were compiled and introduced into the EXFOR database. The measurements originated mainly from five different laboratories: IRMM, Belgium; Forschungszentrum Jülich, Germany; University of Tohoku, Japan; and the University of Debrecen and ATOMKI in Debrecen, Hungary. Example data are shown in figure 2.

Nuclear model calculations were performed using the STAPREH95, STAPRE, EMPIRE-II, TNG and TALYS codes. The studies with TALYS constituted blind calculations with parameters pre-determined from global analyses over the mass table.

Sensitivity coefficients were introduced and determined for selected reactions. The calculated coefficients provide valuable insight into the required uncertainties of the model parameters, if pre-defined target uncertainties for the crosssections are to be met. Graphical inter-comparisons of the experimental data with model calculations have shown that the TALYS default calculations may be an improvement on existing evaluations in about $20 \%$ of cases.

Very large uncertainties can be expected for important reactions when model calculations use a database of parameters that have been determined without reference to measured cross-sections. Thus, a proposal has been made to develop a systematic method for incorporating measured cross-sections and their uncertainties into such parameter databases.

\subsection{Covariance matrix evaluation and processing in the resonance regions}

Uncertainty covariance data are essential for practical applications of evaluated nuclear data, for example in adjustments of group-constants and estimations of design accuracies. A WPEC subgroup was established to study covariance matrix

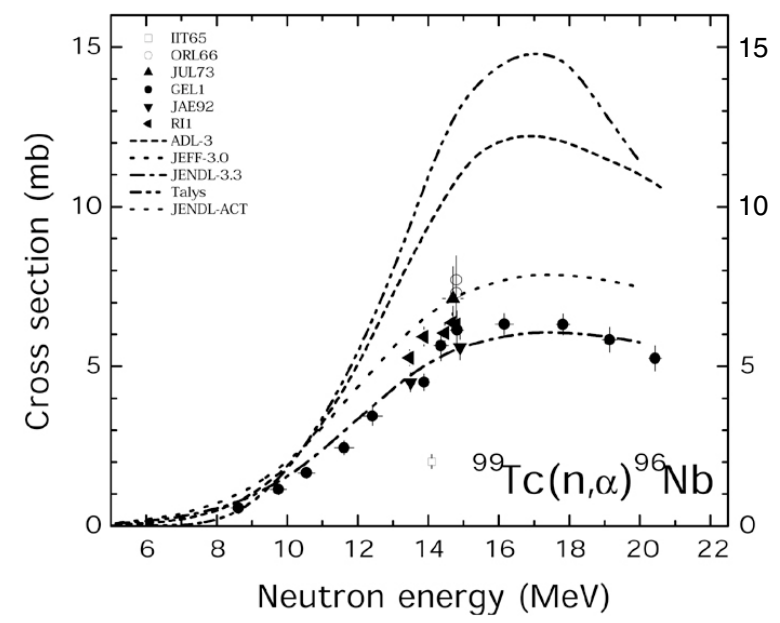

Fig. 2. Examples of measured data obtained at the compact CV cyclotron, FZ Jülich.

evaluation and processing in the resolved and unresolved resonance regions [4].

The subgroup has focused effort on the development of methods to address the following three problems:

- retroactive evaluation of covariance data in the resonance region,

- reduction of large covariance matrices,

- processing of covariance matrices.

A retroactive evaluation technique was used in which experimental data were re-created from the given resonance parameters, and uncertainties in these data were estimated from the experimental conditions. A complete re-evaluation of the basic measurements is thus avoided. The resonance parameter covariance matrix was derived by propagating all these uncertainties from the simulated data to the resonance parameters, i.e., using the R-matrix code to fit the simulated data by varying the resonance parameters. This retroactive method has been implemented in the SAMMY code.

A compact format for storing extremely large covariance matrices was approved in 2004 by the Cross Section Evaluation Working Group (CSEWG), and is now part of the official ENDF-6 format.

As for the processing of covariance data in the resonance region, the subgroup noted that the ERRORJ and the PUFF codes were both able to process Reich-Moore resonances and the compact format cited above.

The retroactive evaluation technique, as well as the compact storage format and processing capabilities, were tested by evaluating the covariance data of the gadolinium isotopes in ENDF/B-VI. The results for the first three resonances of ${ }^{155} \mathrm{Gd}$ at $0.0268,2.008$ and $2.568 \mathrm{eV}$ are shown in figure 3.

\subsection{Fission product cross-sections}

Fission product cross-section data constitute more than $50 \%$ of any evaluated nuclear data library, and these data have not been assessed and revised for many years. The WPEC established a subgroup to review all available evaluations [5], making use of experimental data from the EXFOR library and considering evaluation methodologies. 


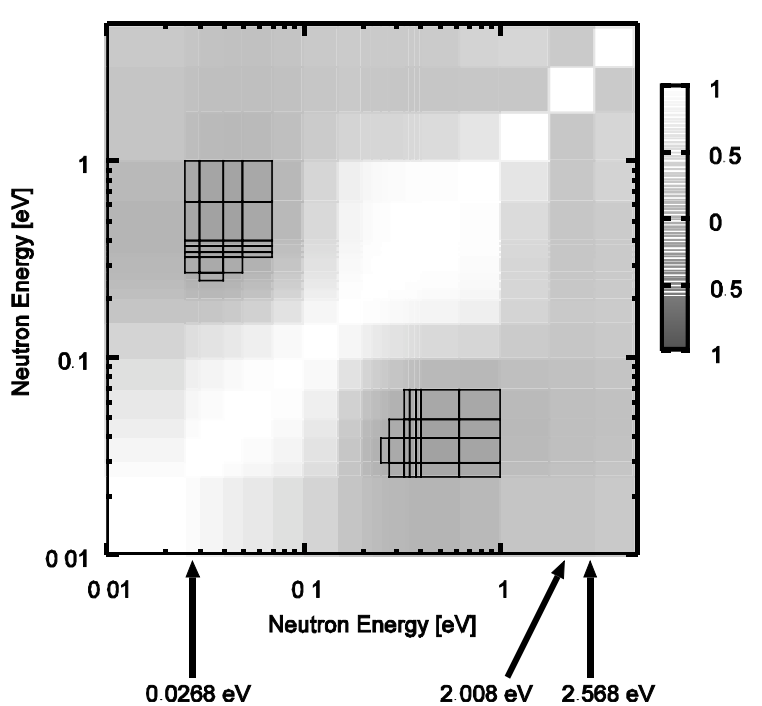

Fig. 3. Correlation matrix of the ${ }^{155} \mathrm{Gd}$ total cross sections in the resonance region - regions surrounded by solid-line boxes have negative correlations.

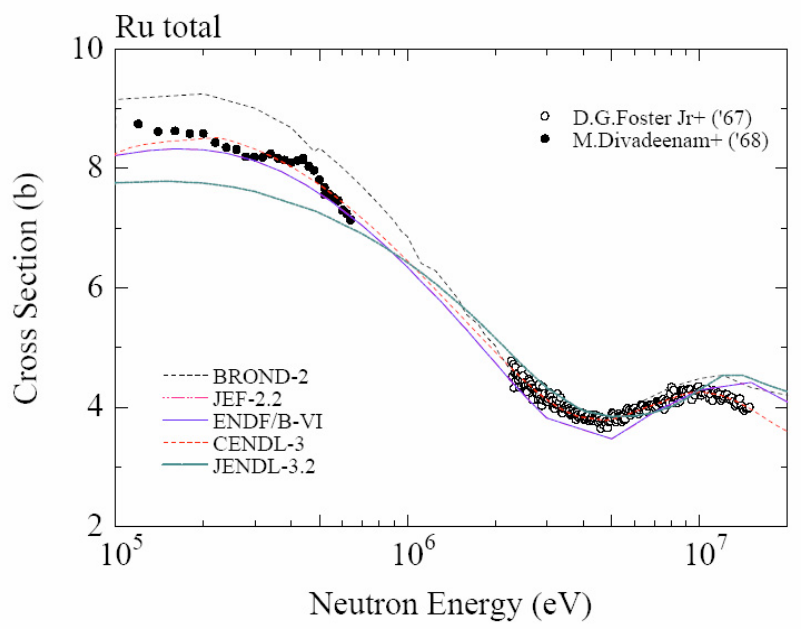

Fig. 4. ${ }^{102} \mathrm{Ru}(\mathrm{n}$,total) cross-sections: evaluated data are compared with experimental data for natural $\mathrm{Ru}$.

A graphical tool was developed to facilitate the visual inter-comparison of the data. Plots were prepared for the most important reaction channels, making altogether nine plots per nucleus and close to 1900 plots in total. An example of such a plot is shown in figure 4.

The adopted review procedure included the following three steps:

- inspection of inter-comparison plots, and the checking of all evaluations and experimental data,

- analysis of thermal cross sections that embraced both the resolved and unresolved resonance regions,

- analysis of the evaluation methodology and consideration of the quality and completeness of the evaluations.

Recommendations were made for 211 isotopes and elements, based on all available material, and proposed evaluations for seven isotopes based on new material. The WPEC has also established a follow-up subgroup to generate and validate a fission product data library based on the resulting recommendations (see below).

\subsection{Nuclear data for improved LEU-LWR reactivity predictions}

A systematic under-prediction of the reactivity of lowenriched uranium-fuelled Light Water Reactor (LWR) systems has been observed when undertaking accurate Monte Carlo transport calculations and the best nuclear data libraries available in 2002. This problem encouraged the WPEC to set up a subgroup to analyse and re-evaluate the nuclear data that impact on the $\mathrm{k}_{\mathrm{eff}}$ of thermal lattices [6]. The isotopes under investigation were ${ }^{235} \mathrm{U},{ }^{238} \mathrm{U},{ }^{16} \mathrm{O}$ and $\mathrm{H}_{2} \mathrm{O}$. Given the recent extensive work devoted to ${ }^{235} \mathrm{U}$ in the resonance range [7] and the good performance of the latest files for highly enriched uranium benchmarks, the work concentrated on ${ }^{238} \mathrm{U}$ and to a lesser extent on ${ }^{16} \mathrm{O}$ and $\mathrm{H}_{2} \mathrm{O}$.

Very close cooperation between reactor physicists and nuclear-data evaluators produced a new evaluation of the ${ }^{238} \mathrm{U}$ thermal capture cross section, the resolved and unresolved resonance parameters and the inelastic scattering data, which provided a credible solution to the reactivity under-prediction problem. Reduced capture in the resolved resonances and a softer secondary inelastic scattering spectrum contributed about equally to the increased reactivity of the new data set (fig. 5). The newer inelastic data are mainly the result of improved theoretical models and more accurate differential measurements.

LANL performed a new evaluation of the ${ }^{16} \mathrm{O}(\mathrm{n}, \alpha)$ reaction that featured a lower $(\mathrm{n}, \alpha)$ cross section. A new evaluation of ${ }^{1} \mathrm{H}-\mathrm{H}_{2} \mathrm{O}$ thermal scattering law data by Mattes et al. was also considered in the study [8].

The results of this work have been incorporated into most of the major evaluated data libraries. The CEA/BRC evaluation of ${ }^{238} \mathrm{U}$ data above the resonance range was

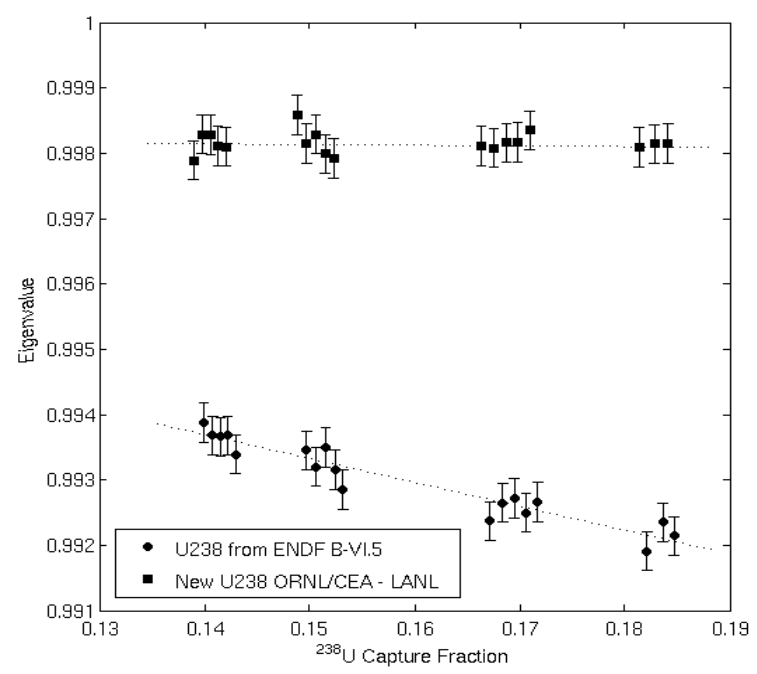

Fig. 5. Calculated eigenvalues of 17 low-enriched uranium benchmarks with ENDF/B-VI.5 and new ${ }^{238} \mathrm{U}$ data. 
Table 2. Radionuclides recommended for TAGS measurements.

\begin{tabular}{lll}
\hline $35-\mathrm{Br}-86$ & $41-\mathrm{Nb}-99$ & $52-\mathrm{Te}-135$ \\
$35-\mathrm{Br}-87$ & $41-\mathrm{Nb}-100$ & $53-\mathrm{I}-136$ \\
$35-\mathrm{Br}-88$ & $41-\mathrm{Nb}-101$ & $53-\mathrm{I}-136 \mathrm{~m}$ \\
$36-\mathrm{Kr}-89$ & $41-\mathrm{Nb}-102$ & $53-\mathrm{I}-137$ \\
$36-\mathrm{Kr}-90$ & $42-\mathrm{Mo}-103$ & $54-\mathrm{Xe}-137$ \\
$37-\mathrm{Rb}-90 \mathrm{~m}$ & $42-\mathrm{Mo}-105$ & $54-\mathrm{Xe}-139$ \\
$37-\mathrm{Rb}-92$ & $43-\mathrm{Tc}-102$ & $54-\mathrm{Xe}-140$ \\
$38-\mathrm{Sr}-89$ & $43-\mathrm{Tc}-103$ & $55-\mathrm{Cs}-142$ \\
$38-\mathrm{Sr}-97$ & $43-\mathrm{Tc}-104$ & $56-\mathrm{Ba}-145$ \\
$39-\mathrm{Y}-96$ & $43-\mathrm{Tc}-105$ & $57-\mathrm{La}-143$ \\
$40-\mathrm{Zr}-99$ & $43-\mathrm{Tc}-106$ & $57-\mathrm{La}-145$ \\
$40-\mathrm{Zr}-100$ & $43-\mathrm{Tc}-107$ & \\
$41-\mathrm{Nb}-98$ & $51-\mathrm{Sb}-132$ & \\
\hline
\end{tabular}

adopted in JEFF-3.1, and the new LANL work on the same isotope was included in ENDF/B-VII.

\subsection{Assessment of fission product decay data}

A WPEC subgroup, comprised of representatives from the decay data evaluation and measurement communities, was formed in response to the fact that different national and international decay data libraries do not generate good estimates of the individual beta (light-particle) and gamma (electromagnetic) components of decay heat, whereas the total decay heat is reasonably well predicted by means of summation calculations. Theoretical decay data have been introduced into the databases with some success, but this approach has proved to be questionable when such data have been replaced with more credible data from other sources. Mean decay energies derived from total absorption gammaray spectroscopy (TAGS) have been shown to assist greatly in the prediction of the component contributions to decay heat, but only limited sets of these experimental data are available.

The following points were proposed in order to make full use of the published TAGS data and improve the reliability of the summation calculations:

1. Identify the nuclides which contribute significantly to FP decay heat over cooling times ranging from 10 to $5000 \mathrm{~s}$.

2. Assess which of these nuclides suffer from inadequately defined decay data due to the "Pandemonium effect".

3 . Identify the need for new $\beta$-feeding measurements by means of TAGS, along with the determination of $\beta^{-}$decay directly to the ground state of the daughter.

Recommendations were made that focus on specific fissionproduct radionuclides that should be measured by TAGS in order to improve decay heat calculations without resorting to theory (see table 2). The resulting WPEC subgroup report will be published later in 2007. Progress made by experimentalists over the course of 2007-2009 will continue to be monitored within the WPEC and IAEA, with the aim of incorporating TAGS-based mean energy data into the existing decay data libraries.

\section{On-going work}

There are presently six WPEC subgroups working on different nuclear data evaluation problems. Two of the subgroups are

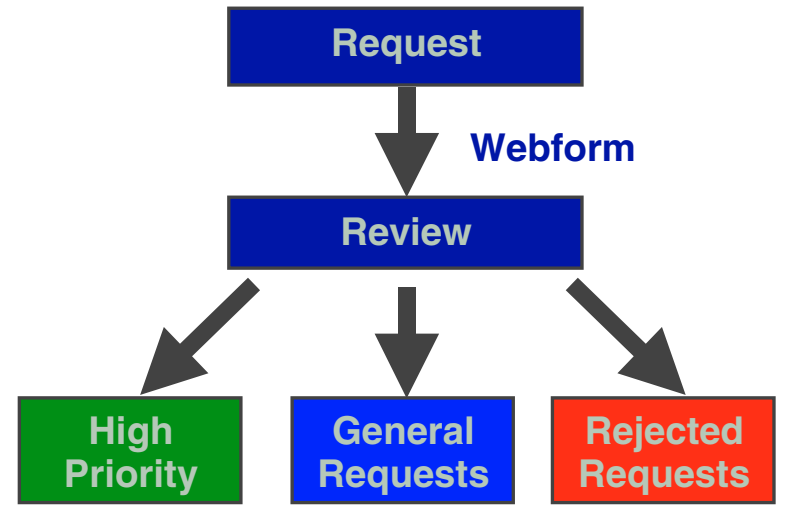

Fig. 6. Procedure for adoption of requests in the NEA High Priority Request List.

developing uncertainty covariance information, two subgroups are reviewing nuclear data needs, and the other two are devoted to fission product data issues.

\subsection{Covariance data}

As a follow-up to the WPEC subgroup on covariance matrix evaluation and processing in the resonance regions (see section 2.3), two new subgroups have been formed.

One of the subgroups is devoted to the fast neutron region, and will develop the capabilities to generate covariances in modern nuclear model codes by means of the Kalman and Monte Carlo sensitivity methods. This subgroup will also address correlations between the resolved and unresolved resonance regions, as well as within the fast neutron region.

The other WPEC subgroup dedicated to data uncertainties will process covariances. A major objective will be to develop the necessary resonance-parameter processing methods, prepare and test new evaluations with resonance-parameter covariance data, and generate cross-section covariance data for sensitivity and uncertainty analyses.

\subsection{Nuclear data requests}

The WPEC has a long-term commitment to maintaining a high priority request list, and a shorter-term activity to identify nuclear data needs for advanced reactor systems.

A few years ago, the WPEC decided to review the High Priority Request List (HPRL) for nuclear data with the aims of improving the acceptance criteria and content. Two lists, will be maintained: "general" requests, and "high priority" requests. The total number of requests in these lists will be reduced significantly by implementing more stringent procedures for acceptance. For example, high priority requests need to be justified by quantitative sensitivity studies and extensive supporting documentation. New procedures for submitting and reviewing requests have also been adopted according to figure 6. More information about the HPRL can be found at the poster session of this conference [9] and on the webpage: www.nea.fr/html/dbdata/hprl/.

Following a workshop on Nuclear Data Needs for Generation-IV Nuclear Energy Systems in Antwerp, Belgium in April 2005 [10], the WPEC established a subgroup to investigate the nuclear data needs for advanced reactor systems. 
Table 3. Sources of 219 evaluations included in the fission product library.

\begin{tabular}{lccc}
\hline $\begin{array}{l}\text { Project } \\
\text { Data Source) }\end{array}$ & $\begin{array}{c}\text { Full } \\
\text { File }\end{array}$ & $\begin{array}{c}\text { Resonance } \\
\text { Region }\end{array}$ & $\begin{array}{c}\text { Fast } \\
\text { Region }\end{array}$ \\
\hline ENDF/B-VI.8 & 1 & 3 & 13 \\
New evals for ENDF/B-VII & 74 & 74 & - \\
JEFF-3.1 & 1 & - & - \\
JENDL-3.3 & 47 & 7 & 56 \\
CENDL-3.0, not yet released & 11 & - & 15 \\
BROND-2.2 & 1 & - & - \\
\hline Total number of materials & $\mathbf{1 3 5}$ & $\mathbf{8 4}$ & $\mathbf{8 4}$ \\
\hline
\end{tabular}

The general objectives of this subgroup are as follows:

- compilation of an agreed set of target accuracies on relevant design parameters for GEN-IV concepts,

- definition of a set of data uncertainties and covariance data,

- production of a set of quantitative data needs based on isotope, reaction type and energy range,

- proposal for an approach to meet the requirements, along with a relative timeframe.

The progress of this WPEC subgroup has been presented at this conference [11].

\subsection{Fission product data}

Following the recommendations concerning fission product cross-sections made by an earlier WPEC subgroup (see section 2.4), a new subgroup has been set up to create and validate a fission product data library based on these recommendations. The creation of a fission product library, containing 219 materials with neutron cross-section data, was completed in 2006, including basic testing with the CHECKR, FIZCON and PSYCHE codes. A complete validation of the data has also been performed using the NJOY-99.161 processing code and neutronics calculations using the MCNP5 Monte Carlo code. Further benchmark tests of the data are underway and some results have been presented at this conference [12]. The origin of the evaluations included in this library is summarized in table 3 .

The second ongoing WPEC activity relates to the lack of reliable prompt photon production data in most of the evaluated nuclear data libraries, especially for fission product data. A subgroup has been established to undertake the following:

- review sources of prompt gammas, mainly from neutron interaction with fission products,

- add the relevant data, especially to the newly created fission product library mentioned above,

- assess the results of sum-up and discrepant point checks from different checking codes.

This work on prompt photon production data is expected to be completed in 2008 .

\section{Future work}

Apart from finalising the work described above, the WPEC has recently approved two new subgroup proposals, which are briefly described below.

\subsection{U-235 capture cross section in the $\mathrm{keV}$ to $\mathrm{MeV}$ energy range}

Recent benchmark analyses in Japan have indicated a possible problem in the capture cross sections of ${ }^{235} \mathrm{U}$ when analysing the BFS and FCA fast-neutron critical experiments that use U fuels. All major evaluated data libraries are unable to describe adequately the sodium voided reactivity and criticalities for these assemblies. The problem is attributable to the large capture cross sections of ${ }^{235} \mathrm{U}$ in the energy region from $100 \mathrm{eV}$ to $2.25 \mathrm{keV}$, over which the same resolved resonance parameters are used in all of the main libraries.

A WPEC subgroup has been established to address this problem by further investigating the origin of the problem from the viewpoint of differential and integral data analyses, and derive recommended ${ }^{235} \mathrm{U}$ capture cross sections in the energy region from about $100 \mathrm{eV}$ to $1 \mathrm{MeV}$.

\subsection{Improvement of accessibility and quality of the EXFOR database}

A subgroup has been established to assess the quality of the EXFOR database of experimental nuclear reaction data and to provide an easy and reliable tool for translating selected parts of the original EXFOR format into an easy accessible tabular form. The correction of compilation mistakes and elimination of errors in EXFOR, as well as the format translating tool, would be beneficial in a number of ways:

- increase the efficient production of evaluated data files,

- easier and more extensive validation of the nuclear model codes.

Any improvements to the EXFOR database will be accomplished through a collaboration between this WPEC subgroup, the International Nuclear Reaction Data Centres Network (NRDC) and EXFOR users, such as nuclear physicists and data evaluators.

\section{Outlook}

A noteworthy trend over the previous decade has been the observed convergence of the major nuclear data libraries in terms of contents, even though they have been assembled independently. One reason for such convergence is the work of WPEC: this working party has identified common problems and initialized common working methods for nuclear data evaluation and, to a lesser extent, validation. Given the limited available manpower, international collaboration to improve the credibility and reliability of recommended nuclear data is essential, and WPEC is expected to remain an important instrument for such evaluations in the years to come.

The authors would like to thank all the contributors to the WPEC reports cited above, and especially the subgroup co-ordinators, A.D. Carlson (subgroup 7), A.J.M. Plompen (subgroup 19 and subgroup C), T. Kawano (subgroup 20), A. Courcelle (subgroup 22), M. Herman (subgroup 24), T. Yoshida (subgroup 25), M. Salvatores (subgroup 26), R. Jacqmin (subgroup 27) and M. Dunn (subgroup 28), who have devoted significant time and effort to organise the work of their subgroups. 


\section{References}

1. A.D. Carlson et al., Nuclear Data Standards, International Evaluation Co-operation, Volume 7, NEA/WPEC-7 (2006).

2. A.D. Carlson et al. (these proceedings).

3. A.J.M. Plompen et al., Neutron activation cross-section measurements from threshold to $20 \mathrm{MeV}$ for the validation of nuclear models and their parameters, International Evaluation Co-operation, Volume 19, NEA/WPEC-19 (2005).

4. T. Kawano et al., Covariance matrix evaluation and processing in the resolved/unresolved resonance regions, International Evaluation Co-operation, Volume 20, NEA/WPEC-20 (2006).

5. P. Oblozinsky et al., Assessment of neutron cross-section evaluations for the bulk of the fission products, International Evaluation Co-operation, Volume 21, NEA/WPEC-21 (2005).
6. A. Courcelle et al., Nuclear data for improved LEU-LWR reactivity predictions, International Evaluation Co-operation, Volume 22, NEA/WPEC-22 (2006).

7. C.R. Lubitz et al., Epithermal capture cross-section of ${ }^{235} \mathrm{U}$, International Evaluation Co-operation, Volume 18, NEA/WPEC18 (1999).

8. M. Mattes, J. Keinert, Thermal neutron scattering data for the moderator materials $\mathrm{H}_{2} \mathrm{O}, \mathrm{D}_{2} \mathrm{O}$ and $\mathrm{ZrH}_{x}$ in ENDF-6 format and as ACE library $M C N P(X)$ codes, IAEA Report INDC(NDS)0470 (2005); see also webpage: http://www-nds.iaea.org/indltsl/.

9. A.J.M. Plompen et al. (these proceedings).

10. Proceedings of the International Workshop on Nuclear Data Needs for Generation IV Nuclear Energy Systems, 5-7 April 2005, Antwerp, Belgium, edited by P. Rullhusen.

11. M. Salvatores (these proceedings).

12. C.J. Dean et al. (these proceedings). 